\title{
IEEE1451 Smart Sensors Architectures for Vital Signs and Motor Activity Monitoring
}

\author{
Octavian Postolache $1, *$ \\ Email opostolache@1x.it.pt \\ Vítor Viegas 2 \\ João Freire 3 \\ José Miguel Dias Pereira 2 \\ Pedro Girão 3 \\ Instituto de Telecomunicações/ISCTE-IUL, Lisboa, Portugal \\ Instituto de Telecomunicações/EST-IPS, Lisboa, Portugal \\ Instituto de Telecomunicações/DEEC-IST, Lisboa, Portugal
}

\begin{abstract}
The chapter presents a set of smart sensing systems for unobtrusive sensing implementations of vital signs and motor activity monitoring compatible with IEEE1451 standard. The IEEE 1451 standard for smart transducers was considered to add smartness to transducers. Regarding the software components of this kind of systems the .NET Framework was considered, taking into account that provides a rich development environment. Thus the implementation of the 1451.1 information model using the .NET Framework, including the customization efforts to meet the requirements of a specific application, which consists, in the present case, of a smart wheelchair prepared to monitor user's physical condition. In one of the implemented architectures the signals from unobtrusive sensors embedded in the wheelchair characterized by plug-and-play and auto-identification capabilities according with IEEE1451.4 are acquired and primary processed at the platform level and transmitted using IEEE802.15.4 wireless communication protocol to a server application implemented in a host PC. Referring to the measurement channels considered in the present approach, are mentioned the photoplethysmography (PPG), skin conductivity (SKC), ballistocardiography (BCG), electrocardiography (ECG) as part of cardiorespiratory activity monitor, while the motor activity is monitored using a $3 \mathrm{D}$ accelerometer.

AQ1
\end{abstract}

\section{Introduction}

In the last years the necessity to reduce the hospitalization costs and to allow proactive and preventative care led to a set of developments in the field of home health monitoring [1-3]. Such systems, denominated vital signs monitors, can include measurement units for blood pressure, heart rate, oxygen saturation [4]. Usually the data from the monitors are sent to a clinical server for early identification of clinical needs, adjustments to the treatment plan and medications, reducing emergency room visits and unplanned hospitalizations. In order to reduce the number of clinical staff needs but also to diminish the stress induced on the patient level caused active participation of the users in measurement setup (e.g. deployment of ECG electrodes) an unobtrusive embedded vital signs monitors associated with daily used objects represent interesting alternative solutions [5]. Our group has been developing a set of smart objects for vital signs and motor activity assessment of elderly people or related to rehabilitation and mobility assessment, as part of different prototypes of smart wheelchairs [6, 7 ] J and smart walkers [ 8, 9]. To assure modular and flexible solutions the vital signs and motor activity, the sensors can be assembled in plug-and-play modules characterized by appropriate conditioning circuits, processing units expressed by low consumption microcontroller and a communication interface. Several solutions regarding "plug-and-play" smart sensors are reported in the literature [ $10-13]$.

Several implementation of 1451 standard for smart transducers is considered special attention being granted to 1451.1 and 1451.4 implementations. One of the smart system architecture makes use 1451.1 information model, which was implemented using the .NET Framework [14]. Several customizations were made to fit the requirements of a specific application in the field of health monitoring. 
The IEEE 1451 std [ 14, 15 ] provides guidelines to build open, high performance and smart transducer systems. It defines a set of neutral communication interfaces for connecting transducers to computers, instrumentation systems and networks. The IEEE 1451 for smart sensors is composed by the following clauses:

- IEEE 1451.0: Introduces the concept of Transducer Interface Module (TIM) as a peripheral that interfaces one or more transducers. The TIM acts as a Data Acquisition (DAQ) board with support for transducer selfidentification, self-diagnosis and self-calibration. The clause defines common functions, communication protocols and Transducer Electronic Data Sheet (TEDS) formats to support these advanced features.

- IEEE 1451.2/3/5: Specify the TIM concept for the cases where the TIM connects to the computer through a set of different communication interfaces.

- IEEE 1451.4: Defines digital wiring, communication protocols and TEDS structures to make analog transducers smarter.

- IEEE 1451.7: Defines communication protocols and TEDS structures to interact with RFID tags.

- IEEE 1451.1 [16]: Defines a generic information model to represent the functionalities of a smart transducer system. The processor that runs the application (and hence implements the information model) is called "Network Capable Application Processor" (NCAP). The information model is composed by three classes of objects: Blocks (intended to process data), Components (intended to store block-related data), and Services (intended to support communications).

The use of commercial development platforms to implement smart transducer interfaces helps to improve market acceptance of the IEEE 1451 standard. A good example is the .NET Framework [ 17, 18], which can be used to implement the 1451.1 information model. This well-known and well-supported framework provides last-generation programming languages (C\# and VB.NET), a high-performance execution environment, and a set of highly productive software libraries. Among these is included the Windows Communication Foundation (WCF) [19], which contains all the stuff needed to develop distributed applications (raw sockets, protocol stacks, web services, etc.).

Following this idea, we implemented a software library—named "Service eXtensions for Instrumentation" (SXI) - that combines the better of two worlds: the communication model provided by WCF and the information model provided by the IEEE 1451.1 clause.

The SXI library was developed strongly related with IEEE 1451 standard and provides guidelines for future developments.

The effectiveness of the SXI library was proven through its usage in the particular field of healthcare monitoring together IEEE1451.4 hardware implementations that is following described.

\section{IEEE 1451.4 Hardware Implementation}

IEEE 1451.4 standard for smart sensors defines a mechanism for adding self-describing behavior to the traditional transducers characterized by analog output signals [20,21], as is the case of the biomedical sensors used for vital signs (ECG, BCG, IPG, PPG) and motor activity monitoring. According with this standard the implemented hardware is based on a microcontroller platform $(\mathrm{uCtrl})$ that allows the acquisition of both analog and digital data from vital signs sensors (Fig. 1). The connectivity between sensors interface modules (SIMi) is assured by a connection board (CB) characterized by a set of 8 sensors and a network controlled circuit (NCC). The SIMi are powered through the power supply line (PWR).

Fig. 1

The block diagram of a microcontroller platform compatible with IEEE 1451.4 for smart sensors embedded in wheelchair (BSi biomedical sensors, $\mathrm{CCi}$-conditioning circuit, ANT-RFID antenna, SIMi sensor interface module, PWR-power supply lines, CB-connection board, MEMi-1-wire EEPROM, NCC-network control circuit) 

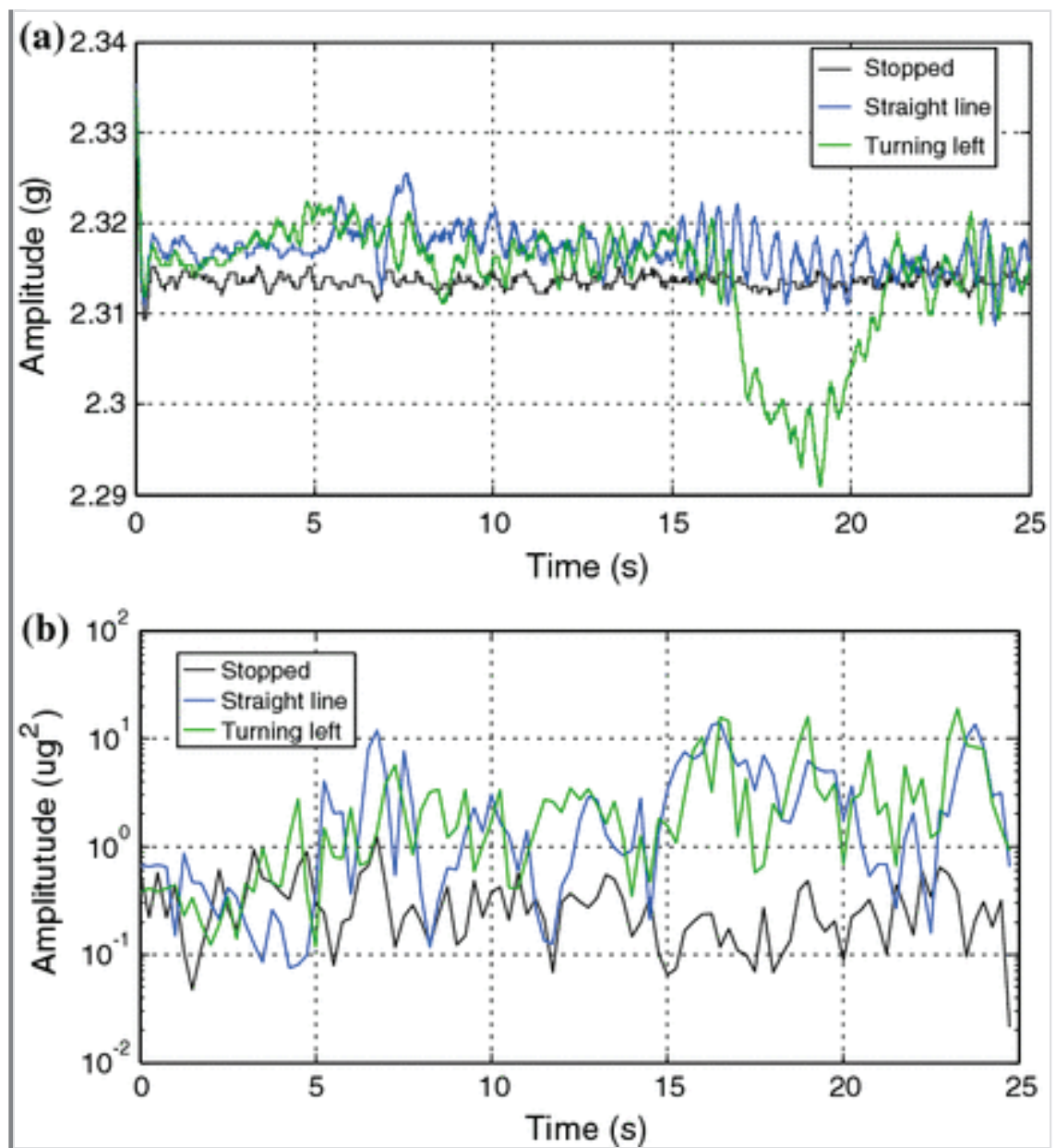

\section{Conclusion}

A set of architectures based on IEEE1451 including hardware and software for vital signs and motor activity of the wheelchairs' users was presented in the chapter. A detailed description of the design and implementation of a microcontroller platform for IEEE 1451.4 is carried out highlighting in particularly elements of hardware expressed by eight measurement channels where the digital information is accessed through 1-wire protocol. The presented IEEE1451 implementations mainly focus on wheelchair user physiological parameters monitoring based on the information acquired through the ECG, PPG, BCG, SKG measurement channels, special attention being granted to kinematic variables (motion activity) monitoring. In one of the hardware implementation physiological variables are sensed by e-textile electrodes embedded on the wheelchair arms, while motion activity is sensed by a 3D MEMS accelerometer. Data acquisition and processing is done onboard the wheelchair by a control station that runs on an embedded PC with a WiFi link. Data monitoring is done remotely by an engineering station installed on a desktop PC. A service extension for instrumentation library is described and the usage for control and engineering stations which implements the 1451.1 information model using WCF services is exemplified. Additionally, smart biomed LabVIEW software, including graphical user interface adapted to the user type was presented, as so as the test results obtained with the presented IEEE1451 implementations. Some tests were done to characterize the sensing channels and to verify the communication capabilities of the SXI library. The obtained results, when compared to reference instruments, showed high performance and good robustness.

\section{References}

. T. Tamura, M. Sekine, Z. Tang, M. Yoshida, Y. Takeuchi, M. Imai, Preliminary study of a new home healthcare monitoring to prevent the recurrence of stroke, in Proceedings of 37th Annual International Conference of the IEEE Engineering in Medicine and Biology Society (EMBC), 2015 (2015), pp. 5489-5492

2. S. Hossain, Patient status monitoring for smart home healthcare, in Proceedings of IEEE International Conference on Multimedia \& Expo Workshops (ICMEW) (2016), pp. 1-6 
3. J. Ma, M. Dong, R\&D of versatile distributed e-home healthcare system for cardiovascular disease monitoring and diagnosis, in Proceedings of International Conference on Biomedical and Health Informatics (BHI) (2014), pp. 444-447

4. O. Postolache, P.M. Girão, H. Ijaz; IEEE 1451.4 embedded smart sensors architecture for wheelchair user monitoring, in Proceedings IEEE International Workshop on Medical Measurements and, vol. 1 (Budapest, Hungary, 2012). pp. 1-5. (May 2012)

5. O.A. Postolache, P.M. Girão, E.C. Pinheiro, J.M. Pereira, R. Madeira, G. Postolache, J.G. Mendes, M.C. Moura, Multi-usage of microwave Doppler radar in pervasive healthcare systems for elderly, in Proceedings Instrumentation and Measurement Technology Conference, I2MTC, 2011 IEEE, (Hangzhou, China, 2011), pp. 1-5. (May, 2011)

6. I. Korhonen, J.E. Bardram, Guest editorial introduction to the special section on pervasive healthcare. IEEE Trans. Inf. Technol. Biomed. 8(3), 229-234 (2004)

7. O. Postolache, P. Girão, J.M. Joaquim, E. Pinheiro, G. Postolache, Physiological parameters measurement based on wheelchair embedded sensors and advanced signal processing. IEEE Trans. Instrum. Meas. 59(10), 2564-2574 (2010)

8. O.A. Postolache, P.M. Girão, E.C. Pinheiro, J.M. Pereira, R. Madeira, G. Postolache, J.G. Mendes, M.C. Moura, Multi-usage of microwave Doppler radar in pervasive healthcare systems for elderly, in Proceedings Instrumentation and Measurement Technology Conference, I2MTC, 2011 IEEE (Hangzhou, China, 2011), pp. 1-5. (May, 2011)

9. O. Postolache, P. Girao, J.M. Dias Pereira, J. Pincho, C. Maia Moura, G. Postolache, Smart walker for pervasive healthcare, in Proceedings of International Conference on Sensing Technology (ICST) (Parlmerston North, NZ, 2011), pp. 1-5. (Dec. 2011)

10. N. Ulivieria, C. Distanteb, T. Lucaa, S. Rocchia, P. Sicilianob, IEEE1451.4: A way to standardize gas sensor. Sens. Actuators B Chem. 114(1), 141-151, 2006. (30 March 2006)

11. National Instruments, An Overview of IEEE 1451.4 Transducer Electronic Data Sheets (TEDS). http://standards.ieee.org/develop/regauth/tut/teds.pdf

12. Futek Inc, Reaction Torque Sensor-Flange/Square Drive IEEE1451.4 compatible. http://www.futek.com/product.aspx?stock=FSH01547\&acc2=accTransducers Techniques, "TEDS IEEE 1451.4 Plug \& Play Smart Sensors”. http://www.transducertechniques.com/plug-and-play-teds.aspx

13. R. Wang, K. Deevy, Reconfigurable adaptive wireless sensor node technology using IEEE 1451.4 standard, in Proceedings of 39th Annual Conference of the IEEE Industrial Electronics Society, IECON 2013 (2013), pp. 3988 $-3993$

14. V. Viegas, J.M. Dias Pereira, P. Silva Girão, A brief tutorial on the IEEE 1451.1 standard. IEEE Instrum. Meas. Mag. 11(2), 38-46 (2008)

15. E.Y. Song, K. Lee, Understanding IEEE 1451-networked smart transducer interface standard. IEEE Instrum. Meas. Mag. 11(2), 11-17 (2008)

16. D.S. Platt, Introducing Microsoft .NET, 3rd ed. (Microsoft Press, WA, USA, 2003). ISBN 9780735619180

17. J. Ritcher, Applied Microsoft .NET Framework Programming (Microsoft Press, WA, USA, 2002). ISBN 9780735614222

18. J. Lowy, Programming WCF Services (O’Reilly, USA, 2007). ISBN 9780596526993

19. J. Smith, Inside Windows Communication Foundation (Microsoft Press, WA, USA, 2007). ISBN 0780735623064

20. K. Lee, Sensor networking and interface standardization, in Proceedings IEEE Instrumentation and Measurement Technology Conference (Budapest, Hungary, 2001), pp. 147-152. (May 21-23, 2001) 
21. P. Doyle, D. Heffernan, D. Duma, A time-triggered transducer network based on an enhanced IEEE 1451 model. Microprocess. Microsyst. 28, 1-12 (2004)

22. R. Madeira, O. Postolache, G. Postolache, Development of therapeutic serious game for a pervasive assitive environment, in IEEE International Conference on Serious Games and Applications for Health-SEGAH (Braga, 2012), pp. 1-11

23. O. Postolache, P. Girao, J.M. Dias Pereira, J. Pincho, C. Maia Moura, G. Postolache, Smart walker for pervasive healthcare, in Proceedings of International Conference on Sensing Technology (ICST) (Parlmerston North, NZ, 2011), pp. 1-5 (Dec. 2011)

24. IEEE Standards, IEEE 1451.4 ${ }^{\mathrm{TM}}$ Manufacturer ID Public Listing. http://standards.ieee.org/develop/regauth/manid/public.html)

25. M.R. Ribeiro, O. Postolache, P.M. Girão, Modular platform architecture for fast prototyping of vital signs and motor activity monitors, in Proceedings IEEE International Instrumentation and Technology Conference I2MTC, vol. 1 (Minneapolis, United States, 2013), pp. 1-6 (May, 2013)

26. Shimmerresearch, A wireless sensor platform for noninvasive biomedical research (2016).

http://www.shimmersensing.com/support/wireless-sensor-networks-papers/ . (November, 2016) 